\title{
Structural Stability and Thermodynamic Properties of (Y2O3)n(n=1-15) Clusters Based on Density Functional Theory
}

\author{
Xin Jiang ${ }^{1}$, Zhenming Zhang ${ }^{1}$, Diqiang Luo ${ }^{1}$, Jinglin You ${ }^{2}$, and Chaobin Lai ${ }^{1}$ \\ ${ }^{1}$ JiangXi University of Science and Technology \\ ${ }^{2}$ Shanghai University
}

May 25, 2021

\begin{abstract}
The initial configuration of Yttrium oxide clusters $(Y 2 O 3) n(n=1-15)$ was creatively constructed by combining artificial bee colony algorithm with density functional theory. The structures of large and medium-sized yttrium oxide clusters with molecular number greater than 10 were established for the first time, and many new structures that are different from existing research have been obtained. The average binding energy, second-order difference energy, HOMO-LUMO gap, density of states and other properties of the clusters were analyzed. The thermodynamic properties and behavior of nano yttrium oxide clusters at different temperatures and sizes were discussed. Studies have shown that for small-sized clusters, the atomic stacking structure is cage-like, while for medium-sized and large-sized clusters, the composite trapezoidal structure and ellipsoid-like structure are more stable. The nanoclusters tend to be stable as a whole, and the relative stability of the cluster structure is higher when $\mathrm{n}=2,4,7,9$. The effect of yttrium oxygen atomic orbital on bonding is analyzed. The heat capacity (Cp), enthalpy change $(\mathrm{H})$ and entropy $(\mathrm{S})$ of $(\mathrm{Y} 2 \mathrm{O} 3) \mathrm{n}(\mathrm{n}=1-15)$ clusters increase with the increase of temperature $(\mathrm{T})$, and the vibration free energy (Gv) decreases with the increase of $\mathrm{T}$. The stability of the clusters changes in the temperature range of $300 \mathrm{~K}-500 \mathrm{~K}$.
\end{abstract}

\section{Structural Stability and Thermodynamic Properties of $\left(\mathrm{Y}_{2} \mathrm{O}_{3}\right)_{n}(\mathrm{n}=1-15)$ Clusters Based on Density Functional Theory}

Xin Jiang ${ }^{1}$, Zhenming Zhang ${ }^{1}$, Diqiang Luo ${ }^{1}$, Jinglin You $^{2}$, Chaobin Lai $^{1}$

${ }^{1}$ Faculty of Materials Metallurgy and Chemistry,JiangXi university of science and technology,GanZhou,China

${ }^{2}$ ShangHai university,ShangHai,China

\section{Correspondence}

Chaobin Lai,Faculty of Materials Metallurgy and Chemistry,JiangXi university of science and technology,GanZhou,China

\section{Email:lcb5115@126.com}

Abstract :The initial configuration of Yttrium oxide clusters $\left(\mathrm{Y}_{2} \mathrm{O}_{3}\right)_{n}(\mathrm{n}=1-15)$ was creatively constructed by combining artificial bee colony algorithm with density functional theory. The structures of large and medium-sized yttrium oxide clusters with molecular number greater than 10 were established for the first time, and many new structures that are different from existing research have been obtained. The average binding energy, second-order difference energy, HOMO-LUMO gap, density of states and other properties of the clusters were analyzed. The thermodynamic properties and behavior of nano yttrium oxide clusters at different temperatures and sizes were discussed. Studies have shown that for small-sized clusters, the atomic stacking structure is cage-like, while for medium-sized and large-sized clusters, the composite trapezoidal structure and ellipsoid-like structure are more stable. The nanoclusters tend to be stable as a whole, and 
the relative stability of the cluster structure is higher when $\mathrm{n}=2,4,7,9$. The effect of yttrium oxygen atomic orbital on bonding is analyzed. The heat capacity $\left(\mathrm{C}_{\mathrm{p}}\right)$, enthalpy change $(\mathrm{H})$ and entropy $(\mathrm{S})$ of $\left(\mathrm{Y}_{2} \mathrm{O}_{3}\right)_{\mathrm{n}}$ $(\mathrm{n}=1-15)$ clusters increase with the increase of temperature $(\mathrm{T})$, and the vibration free energy $\left(\mathrm{G}_{\mathrm{V}}\right)$ decreases with the increase of $\mathrm{T}$. The stability of the clusters changes in the temperature range of $300 \mathrm{~K}-500 \mathrm{~K}$.

Keywords: Nanocluster structure; Density functional theory; Structural stability;Thermodynamic properties;ABC algorithm

\section{Introduction}

Rare earth elements have many unique properties. Adding trace rare earth elements into metal materials can improve the microstructure, mechanical properties, oxidation resistance and corrosion resistance of metals to varying degrees. The results show that the addition of rare earth elements in the smelting process of metals can play a role in purifying, modifying inclusions and microalloying ${ }^{[1-6]}$. These effects will change the morphology and distribution of inclusions in metals, so that more inclusions with very small particle diameter and dispersed distribution are generated in the solidification process of metals, and then improve their comprehensive properties. At present, the mechanism of rare earth's morphology and action form in metals is not clear, which affects its efficient utilization. As a transition state between solid state and gas state, the emergence of cluster research provides a suitable research method for the development and improvement of the theory of atomic bonding, the existence form and the formation law of various macromolecules.

Yttrium oxide $\left(\mathrm{Y}_{2} \mathrm{O}_{3}\right)$ is a deoxidation product of rare earth treatment of molten steel. It is a typical rare earth oxide and has the general properties of rare earth oxides. It has a wide range of technical applications in the fields of electronics, optics, mechanical engineering, metallurgical engineering and catalyst support ${ }^{[7]}$. At room temperature, yttrium oxide has a C-type cubic structure of rare earth oxides, belonging to the bodycentered cubic structure of iron manganese ore type (space group Ia3), similar to fluorite $\left(\mathrm{CaF}_{2}\right)$ structure with a quarter anion vacancy, and the band gap is $5.8 \mathrm{eV}^{[8-10]}$. Yttrium oxide cluster is a kind of transition state and intermediate product in the nucleation of yttrium oxide crystal. At present, most of these studies restricted to the small size with monomer, dioxide, and trioxide clusters ${ }^{[11-14]}$. In the experiment, Wu and Wang ${ }^{[15]}$ studied the electronic structure of small $\mathrm{YO}_{\mathrm{n}}{ }^{-}$clusters with $\mathrm{n}=1-5$ by photoelectron spectroscopy (PES), obtained the vibration-resolved photoelectron spectroscopy of $\mathrm{YO}_{\mathrm{n}}{ }^{-}$, and measured the electron affinity of YO. Pramann ${ }^{[16]}$ et al. studied the electron affinity and vertical ionization energy of $\mathrm{Y}_{\mathrm{n}} \mathrm{O}_{\mathrm{m}}{ }^{-}$ clusters with $n=2-10, m=1-3$, discussed the evolution process of the electronic structure of neutral yttrium oxide clusters, and compared the difference of yttrium oxide dissociation energy. Knickelbein ${ }^{[17]}$ calculated the photoelectric energy spectra of $Y_{n}$ and $Y_{n} O$ clusters with $n=2-31$, and studied the variation law of their vertical ionization energy. In terms of theoretical calculations, Amol B. Rahane ${ }^{[18]}$ et al. studied smallsized yttrium oxide clusters with molecular number of 1-10, and discussed their stability and electronic characteristics. Rong Li and Qiyao Zhang ${ }^{[19,20]}$ studied the structure and stability of $\left(\mathrm{Al}_{2} \mathrm{O}_{3}\right)_{\mathrm{n}}(\mathrm{n}=7$ and $\mathrm{n}=15)$, and obtained many isomers. Xiao Jianyun ${ }^{[21]}$ et al. studied $(\operatorname{HgSe})_{n}(n=1-6)$ clusters using density functional theory, obtained the equilibrium geometry, vertical ionization energy results, and analyzed the atomic net charge distribution, frontier molecular orbital characteristics.

Previous studies only studied the structure and photoelectric energy spectrum of yttrium oxide ions or single element yttrium, and discussed the effects of different oxygen to metal ratios on their structure and electronic properties. However, there were few studies on the neutral $\mathrm{Y}_{2} \mathrm{O}_{3}$ clusters structure, especially the cluster structure with medium and large sizes. In this paper, the structure and properties of yttrium oxide clusters are studied by artificial bee colony algorithm combined with quantum chemical calculation, in order to improve the thermodynamic data of nano yttrium oxide clusters, and lay the foundation for exploring the nucleation process of rare earth inclusions in metal materials, and provide technical guidance for the process of adding rare earth yttrium in metal and the size control of inclusions. 


\section{Computational details}

The molecular orbital theory program based on density functional theory (DFT) in the Gaussian $09^{[22]}$ software package was used to optimize the structure and calculate the thermodynamic properties. First, use the ABC algorithm ${ }^{[23-25]}$ (artificial bee colony algorithm) to construct the initial configuration of $\left(\mathrm{Y}_{2} \mathrm{O}_{3}\right)_{\mathrm{n}}(\mathrm{n}=1$ 15), that is, set up different boxes for different system sizes, and add a certain number of oxygen atoms and yttrium atoms inside,automatically generates a large number of initial configurations according to the internal algorithm of the program. Then, preliminary optimization is carried out under the semi-empirical method, and the more stable partial structures are screened out according to the energy level of dozens to thousands of calculated structures for the next step of precise calculation and optimization. In order to ensure the accuracy of the ground state structure, on the basis of fully considering the spin multiplicity, for each cluster size, as many initial structures as possible are selected for geometric optimization. Finally, the global optimal structure is obtained and the frequency calculation is performed to obtain various thermodynamic properties of the cluster. In the precise calculation, the BP86 functional and mixed basis set are used, that is, the all-electronic basis set $6-311 \mathrm{G}^{*}$ is used for oxygen elements. For the heavier rare earth yttrium element, the Stuttgart pseudopotential and the corresponding pseudopotential are used considering the relativistic effect ${ }^{[26-28]}$. Finally, the optimal structure of different isomers with $n=1-15$ and the structure with lower energy are obtained.

\section{Results and discussion}

\section{Geometry structures}

Using the method of combining ABC and DFT, a series of structures of $\left(\mathrm{Y}_{2} \mathrm{O}_{3}\right)_{n}(\mathrm{n}=1-15)$ were obtained. When $\mathrm{n}=1-5$, most of the global optimal structures obtained are consistent with existing reports, but when $\mathrm{n}=6-15$, many new stable structures have been searched out. These structures will be discussed in detail below.

$\mathrm{n}=1-5$

Figure 1 shows the global optimum and low energy structure of $\left(\mathrm{Y}_{2} \mathrm{O}_{3}\right)_{n}(\mathrm{n}=1-5)$ clusters, where red is oxygen atom and blue is yttrium atom. As shown in Figure 1, when $n=1$, the global optimal structure of $\mathrm{Y}_{2} \mathrm{O}_{3}$ is symmetrical tetrahedron, $\mathrm{D}_{3 \mathrm{~h}}$ symmetry, kite-like $1 \mathrm{~B}$ structure is $1.56 \mathrm{ev}$ higher than the optimal structure, and its spatial configuration is the global $\mathrm{Al}_{2} \mathrm{O}_{3}$ optimal structure ${ }^{[20]}$. The $1 \mathrm{C}$ linear structure is 4.1ev higher in energy than $1 \mathrm{~A}$, and the $\mathrm{Y}-\mathrm{O}$ bond is shorter.For $\left(\mathrm{Y}_{2} \mathrm{O}_{3}\right)_{2}$, the global optimal structure is a cage-like structure $(2 \mathrm{~A})$ with $\mathrm{T}_{\mathrm{d}}$ symmetry, this configuration is also the optimal structure of $\left(\mathrm{Al}_{2} \mathrm{O}_{3}\right)_{2}{ }^{[19]}$. The $2 \mathrm{~B}$ structure is $1.56 \mathrm{ev}$ higher in energy than the $2 \mathrm{~A}$ structure. 4 oxygen atoms and 2 yttrium atoms form a plane, and are connected with $2 \mathrm{YO}$ units. This configuration is a relatively stable structure of $\left(\mathrm{Fe}_{2} \mathrm{O}_{3}\right)_{2}{ }^{[29]}$. When $\mathrm{n}=3$, five different structures are given, and the cap structure shown in $3 \mathrm{~A}$ is considered to be the global optimal structure with $\mathrm{C}_{1}$ symmetry. The structure of $3 \mathrm{~B}$ is similar to that of $3 \mathrm{~A}$, only $0.07 \mathrm{ev}$ higher in energy than the latter, with $\mathrm{C}_{\mathrm{S}}$ symmetry. The structures of $3 \mathrm{C}, 3 \mathrm{D}$ and $3 \mathrm{E}$ have been reported in previous studies of alumina clusters. $4 \mathrm{~A}$ is a newly discovered structure with $\mathrm{C}_{1}$ symmetry and the lowest energy among all configurations. The 4B structure has also been studied by others. They believe that the optimal structure is $0.41 \mathrm{ev}$ lower in energy than the $4 \mathrm{~B}$ structure, while $4 \mathrm{~A}$ is $0.64 \mathrm{ev}$ lower than $4 \mathrm{~B}$. Therefore, the newly discovered $4 \mathrm{~A}$ structure can be considered as the global optimal structure. Compared with others, the $5 \mathrm{~A}$ structure has lower electron energy and $\mathrm{C}_{2 \mathrm{v}}$ symmetry, which is consistent with the results obtained by Amol B. Rahane ${ }^{[18]}$ et al. 5B, 5C, and $5 \mathrm{E}$ are similar to the alumina cluster structure reported by RongLi ${ }^{[20]}$ et al. 


\section{$\mathrm{n}=6-10$}

For medium-sized clusters, the spatial structure is more complex. Figure 2 shows the global optimum and lower energy structure diagram of $\left(\mathrm{Y}_{2} \mathrm{O}_{3}\right)_{n}(\mathrm{n}=6-10)$ clusters. When $\mathrm{n}=6$, the global optimal structure of $\left(\mathrm{Y}_{2} \mathrm{O}_{3}\right)_{6}$ is a ladder structure with $\mathrm{C}_{\mathrm{S}}$ symmetry, which is different from the research results of Amol B. Rahane $^{[18]}$ et al. The structures of $6 \mathrm{~B}$ and $6 \mathrm{C}$ are relatively similar, and the energy difference is only 0.22 energy from $6 \mathrm{~A}$. $6 \mathrm{D}$ and $6 \mathrm{E}$ are newly discovered structures. For $\left(\mathrm{Y}_{2} \mathrm{O}_{3}\right)_{7}$, we consider that $7 \mathrm{~A}$ shows the optimal structure, $\mathrm{C}_{S}$ symmetry, which is different from the results reported by Amol B. Rahane ${ }^{[18]}$. $7 \mathrm{~B}$ is a newly searched configuration with cage structure and $\mathrm{C}_{1}$ symmetry, which is $0.06 \mathrm{ev}$ higher than $7 \mathrm{~A}$ in energy. 7C, 7D and 7E are similar to 7B in atomic stack, but the energy is higher than that of the latter. When $\mathrm{n}=8$, the global optimal structure is a pyramid-like structure, which has $\mathrm{C}_{\mathrm{S}}$ symmetry. The structures of $8 \mathrm{~B}, 8 \mathrm{D}$ and $8 \mathrm{E}$ are similar, which is consistent with the partial structure of $\left(\mathrm{Al}_{2} \mathrm{O}_{3}\right)_{8}{ }^{[20]}$. $8 \mathrm{C}$ is a new structure, $\mathrm{C}_{\mathrm{S}}$ symmetry, energy is $0.2 \mathrm{ev}$ higher than the optimal structure. The global optimal structure shown in $9 \mathrm{~A}$ is similar to the alumina cluster studied by Qiyao Zhang ${ }^{[19]}$ et al., which has $\mathrm{C}_{1}$ symmetry and the lowest electron energy. The structures such as $9 \mathrm{~B}$ and $9 \mathrm{C}$ are similar to $9 \mathrm{~A}$, and the energy difference is small. When $\mathrm{n}=10$, the $10 \mathrm{~A}$ with $\mathrm{C}_{60}$-like structure is the global optimal structure, which consists of 19 yttrium atoms and 30 oxygen atoms forming a space sphere-like surface with one yttrium atom wrapped inside. This is a newly discovered configuration. The first five structures with the lowest energy in global search are all spherical structures.

\section{$\mathrm{n}=11-15$}

For larger clusters, the system gradually evolves into ellipsoidal structure. Fig. 3 shows the schematic diagram of the global optimum and low energy structure of $\left(\mathrm{Y}_{2} \mathrm{O}_{3}\right)_{n}(\mathrm{n}=11-15)$ clusters, which is the first calculated large $\mathrm{Y}_{2} \mathrm{O}_{3}$ cluster structure. For $\left(\mathrm{Y}_{2} \mathrm{O}_{3}\right)_{11}$, the tower-shaped spatial three-dimensional structure is the global optimal structure, which evolves from 10A and has $\mathrm{C}_{\mathrm{S}}$ symmetry, which has the same spatial structure as $\left(\mathrm{Al}_{2} \mathrm{O}_{3}\right)_{11}$ reported by Qiyao Zhang ${ }^{[19]}$ et al. The other isomers are similar in structure but slightly higher in energy. When $\mathrm{n}=12$, the global optimal structure of the system is ellipsoidal (12A), which has $\mathrm{C}_{\mathrm{S}}$ symmetry. The hollow ellipsoidal surface is formed by 22 yttrium atoms and 31 oxygen atoms. The interior contains two yttrium atoms and five oxygen atoms, and the distance between five oxygen atoms and the central yttrium atom is equal. The other structures $(12 \mathrm{~B}, 12 \mathrm{C}, 12 \mathrm{D}$, etc.) also have similar spatial configurations, but the energy is higher than that of $12 \mathrm{~A}$. When $\mathrm{n}=13$, the optimal structure (13A) evolves from $12 \mathrm{~A}$, which also has ellipsoidal structure and $\mathrm{C}_{\mathrm{S}}$ symmetry. The extra two yttrium atoms and three oxygen atoms are distributed inside the ellipsoidal surface and the surface, respectively. For $\left(\mathrm{Y}_{2} \mathrm{O}_{3}\right)_{14}$, we believe that $14 \mathrm{~A}$ is the global optimal structure, which is a spherical structure with slightly flat ends and slightly bulging middle, and has $\mathrm{C}_{1}$ symmetry. The $14 \mathrm{~A}$ configuration has atomic stratification, and there are 11 layers of atoms from top to bottom and they are symmetrical. Through the comparison of energy, it is found that this configuration has high stability. 14B has similar spatial configurations.Finally, when $\mathrm{n}=15$, the global optimal structure is shown in $15 \mathrm{~A}$, and a $\mathrm{Y}$ atom connects three oxygen atoms from the ellipsoid body, which is different from the above structure. 15B, $15 \mathrm{C}$ have similar configurations.

\section{Structural stability}

The average binding energy $\mathrm{E}_{\mathrm{b}}$ of the cluster is usually used to characterize the stability of the cluster structure. The average binding energy $\mathrm{E}_{\mathrm{b}}$ of the cluster reflects the energy released by each atom in the cluster. The larger the average binding energy is, the more stable the system is. The calculation method of $\mathrm{E}_{\mathrm{b}}$ is as follows :

$\mathrm{E}(\mathrm{Y}), \mathrm{E}(\mathrm{O})$ and $\mathrm{E}\left(\mathrm{Y}_{2} \mathrm{O}_{3}\right)_{\mathrm{n}}$ represent the total energies of single yttrium, oxygen atoms and clusters, respectively.

Fig. 4 shows the change trend of binding energy $E_{b}$ of $\left(\mathrm{Y}_{2} \mathrm{O}_{3}\right)_{n}(\mathrm{n}=1-15)$ clusters and $\mathrm{Y}_{\mathrm{n}}{ }^{[30]}$ clusters with the increase of cluster size.It can be seen from Figure 4 that the average binding energy of $\left(\mathrm{Y}_{2} \mathrm{O}_{3}\right)_{n}(n=1-15)$ 
clusters increases with the increase of cluster size, and gradually tends to be saturated. From the perspective of growth trend, the growth rate of yttrium oxide clusters is close to that of $Y_{n}$ clusters in the growth process, and the trends of the two are similar. Compared with $Y_{n}$ clusters, the average binding energy of yttrium oxide clusters increased more obviously at the initial stage of the cluster system. This is mainly due to the fact that the valence bond orbital of oxygen atom is less than half full $\left(2 \mathrm{P}^{4}\right)$, while the valence bond orbital of yttrium atom is full $\left(5 \mathrm{~S}^{2}\right)$, and the probability of electron filling p orbital is much larger than that of filling $\mathrm{s}$ orbital. Therefore, it shows that the average binding energy growth trend of yttrium clusters is slower than that of mixed clusters. With the increase of the system, the average binding energy of yttrium oxide clusters increases slowly and gradually tends to saturation, which is similar to that of pure yttrium clusters. This is because the coordination number of all atoms is almost saturated, the contribution to the binding energy decreases, and the average binding energy becomes flat. In addition, the average binding energy of mixed clusters is larger than that of pure yttrium clusters, which indicates that the stability of doped clusters is higher than that of pure yttrium clusters.

Second-order energy difference $\Delta^{2} \mathrm{E}$ is a sensitive physical quantity to judge the relative stability of clusters in cluster physics.In the experiment, the fine structure of the cluster mass spectrum distribution mainly depends on the difference between the binding energy of the two adjacent clusters, and the relatively stable cluster corresponds to the peak position of the mass spectrum ${ }^{[31,32]}$. Therefore, the relative stability of the cluster structure corresponds to the second-order energy difference, and the larger the difference score is, the higher the stability is compared with the adjacent clusters. The calculation formula is as follows :

where $E_{\text {total }}(n+1)$ indicates that the system increases the total energy of an atom, $E_{\text {total }}(n-1)$ indicates that the system reduces the total energy of an atom, and $E_{\text {total }}(n)$ indicates the total energy of the system. The energy levels of the highest occupied orbital (HOMO) and the lowest space orbital (LUMO) can reflect the strength of the electron gain and loss ability of the cluster molecules, HOMO-LUMO energy gap is an important parameter to characterize the electronic structure and stability of clusters. The size of HOMOLUMO energy gap reflects the ability of electrons to transition from occupied orbit to empty orbit, which represents the ability of molecules to participate in chemical reactions to a certain extent. Higher energy gap means that higher energy is needed to change the electronic structure of clusters, so clusters have higher stability and lower chemical properties.

Figure 5 shows the trend of second-order energy difference $\Delta^{2} \mathrm{E}$ and H-L energy gap with the increase of clusters size. It can be seen that with the increase of the number of atoms, the second-order difference energy changes alternately. When the number of atoms in the system is $10,20,35,45,60$, it is at the peak, indicating that these structures have strong structural stability relative to the nearby size. When the number of atoms is 10, the curve reaches the maximum, indicating that $\left(\mathrm{Y}_{2} \mathrm{O}_{3}\right)_{2}$ has the highest stability in the whole cluster evolution process. It can be seen from the H-L energy gap curve that when the cluster size is in the range of 5-55 atoms, the energy gap decreases with the increase of the total atomic number of the cluster. When the atomic number is greater than or equal to 55, the H-L energy gap fluctuates. It can be seen from the above analysis that the change trend of the second-order energy difference $\Delta^{2} \mathrm{E}$ and the $\mathrm{H}-\mathrm{L}$ energy gap is generally consistent.

In order to further analyze the contribution of different elements in the cluster system to bond formation, the molecular orbital composition of $\left(\mathrm{Y}_{2} \mathrm{O}_{3}\right)_{n}(\mathrm{n}=1-15)$ clusters was analyzed by using the wave function analysis program Multiwfn ${ }^{[2,33]}$. Fig. 6 shows the schematic diagram of the total state density (TDOS), local state density (PDOS, which reflects the contribution curve of specific fragments to TDOS) and overlapping population state density (OPDOS) of $\left(\mathrm{Y}_{2} \mathrm{O}_{3}\right)_{14}$. Discrete lines represent original data, the curves broadened from which have been scaled by factor of 0.03 . Left axis is for total and partial density of states, right axis is for overlap density of states. Notice that only relative rather absolute height of curves is meaningful. The graph clearly exhibits orbital characteristics in different energy ranges. It is obvious that the major contribution from $\mathrm{S}$ basis function of oxygen (magenta curve) is due to low-lying MOs instead of frontier MOs. The major compositions of MOs around $-7.00 \mathrm{eV}$ are $\mathrm{P}_{\mathrm{x}}, \mathrm{P}_{\mathrm{y}}$ and $\mathrm{P}_{\mathrm{z}}$ orbitals of oxygen (blue curve) and yttrium atom (red curve). Inspection of the green OPDOS curve, which expresses the bonding between oxygen $\mathrm{P}_{\mathrm{x}}, \mathrm{P}_{\mathrm{y}}, \mathrm{P}_{\mathrm{z}}$ 
and yttrium atom, suggests that oxygen $\mathrm{P}_{\mathrm{x}}, \mathrm{P}_{\mathrm{y}}$ and $\mathrm{P}_{\mathrm{z}}$ orbitals are important for stabilization of $\left(\mathrm{Y}_{2} \mathrm{O}_{3}\right)_{14}$. HOMO is almost purely contributed by yttrium orbitals. For all virtual MOs, OPDOS curve is in negative region and show antibonding characteristic. This is due to the unfavorable overlapping in orbital phase,as can been seen from LUMO isosurface.

\section{Thermodynamic property}

In order to further analyze the influence of the structure and size of nanoclusters on their thermodynamic properties, gaussian09 was used to calculate the frequency of yttrium oxide $\left(\mathrm{Y}_{2} \mathrm{O}_{3}\right)_{\mathrm{n}}(\mathrm{n}=1-15)$, and the thermodynamic data of different sizes at $0-2000 \mathrm{~K}$ were obtained by thermodynamic analysis software Shermo ${ }^{[34]}$. The results are shown in Figure 1. The calculated energy $\mathrm{E}_{0}$ of yttrium oxide clusters at $0 \mathrm{~K}$ is shown in table 1.

Fig.7(a)(c) shows the variation of the heat capacity $\left(\mathrm{C}_{\mathrm{p}}\right)$ and entropy $(\mathrm{S})$ of the calculated nanocluster $\left(\mathrm{Y}_{2} \mathrm{O}_{3}\right)_{\mathrm{n}}(\mathrm{n}=1-15)$ with temperature $(\mathrm{T})$ and the molecular number $\mathrm{n}$ of the clusters. It can be seen that in the temperature range of 0-2000 K, at the same temperature, the heat capacity and entropy increase with the increase of $n$; For clusters with the same $n$ value, the heat capacity and entropy increase with increasing temperature. In addition, the change rate of the two decreases with the increase of temperature, that is, in the low temperature stage, the change is faster with the increase of temperature. In the high temperature stage, the increase rate decreases with the increase of temperature, and finally tends to be constant. It can be seen that the variation law of heat capacity and entropy of $\left(\mathrm{Y}_{2} \mathrm{O}_{3}\right)_{n}$ with temperature is consistent with that of conventional materials, but the two change with $\mathrm{n}$ (molecular number, representing different structures and also changing the size to a certain extent), which reflects the influence of structure and size on thermodynamic properties.

Fig.7(b) shows the variation of the enthalpy $(\mathrm{H})$ of nanoclusters $\left(\mathrm{Y}_{2} \mathrm{O}_{3}\right)_{n}(\mathrm{n}=1-15)$ with temperature $(\mathrm{T})$ and clusters size. In the temperature range of $0-2000 \mathrm{~K}$, at the same temperature, $\mathrm{H}$ increases with the increase of $\mathrm{n}$; For clusters with the same $\mathrm{n}$ value, $\mathrm{H}$ also tends to increase with the increase of temperature. In addition, the rate of change of $\mathrm{H}$ with temperature gradually increases with the increase of temperature. That is, in the low temperature stage, the heat capacity changes slowly with the increase in temperature, and in the higher temperature stage, the increase in the heat capacity gradually increases with the increase in temperature.

Fig.7(d) shows the variation of vibrational free energy $\left(G_{v}\right)$ of nanoclusters $\left(\mathrm{Y}_{2} \mathrm{O}_{3}\right)_{n}(\mathrm{n}=1-15)$ with temperature $(\mathrm{T})$ and clusters size. The internal amplification diagram is the variation law in the temperature range of 300-500K. In this temperature range, the $\mathrm{G}_{\mathrm{v}}$ curves of different clusters are pairwise intersecting. It can be seen that when the temperature is below $350 \mathrm{~K}$, at the same temperature, $\mathrm{G}_{\mathrm{v}}$ increases with the increase of $\mathrm{n}$; When the temperature is higher than $450 \mathrm{~K}, \mathrm{G}_{\mathrm{v}}$ decreases with increasing $\mathrm{n}$ at the same temperature. For clusters with the same $n$ value, $G_{v}$ decreases with increasing temperature. In addition, the change rate of $G_{v}$ with temperature of clusters with larger $n$ value is greater than that of clusters with smaller $n$ value. In the low temperature stage, the $G_{v}$ of large clusters is larger than that of small clusters, but with the increase of temperature, the $G_{v}$ of large clusters decreases with the increase of temperature. In the high temperature stage, the $G_{v}$ of large clusters is lower than that of small clusters. In general, the variation of $G_{v}$ with temperature of $\left(\mathrm{Y}_{2} \mathrm{O}_{3}\right)_{\mathrm{n}}(\mathrm{n}=1-15)$ clusters is consistent with that of macroscopic thermodynamics, but the special law of vibrational free energy with temperature reflects the inconsistency of structural stability of large and small clusters with temperature, which is completely different from macroscopic materials and is the embodiment of special thermodynamic properties of nanoclusters.

Fig.7(e) shows the variation of absolute Gibbs free energy $(\mathrm{G})$ of nanoclusters $\left(\mathrm{Y}_{2} \mathrm{O}_{3}\right)_{\mathrm{n}}(\mathrm{n}=1-15)$ with temperature $(T)$ and molecular number $n, G=G_{v}+E(0 K)$. It can be seen from the graph that $\mathrm{G}$ decreases with the increase of $\mathrm{n}$ at the same temperature from 0 to $2000 \mathrm{~K}$. For clusters with the same $\mathrm{n}$ value, the change trend of $G$ is not obvious with the increase of temperature, which is mainly because for a cluster with a $n$ value, its $E(0 K)$ is far lower than its $G_{v}$ value, so the size of $G$ is mainly determined by the size of $E(0 K)$, 
and $\mathrm{G}_{\mathrm{v}}$ only has a certain influence on $\mathrm{G}$. Therefore, the $\mathrm{G}$ curves of different clusters are almost parallel, but actually not parallel. The slope is caused by the $G_{v}$ value of different clusters. It can also be seen from the absolute Gibbs free energy that the structure of the cluster plays a decisive role in its thermodynamic properties. Thermodynamic properties of nano yttrium oxide clusters are similar to those of alumina clusters studied by Guo Cheng Wang ${ }^{[35,36]}$ et al.

The regression equations of thermodynamic properties of $\left(\mathrm{Y}_{2} \mathrm{O}_{3}\right)_{n}(\mathrm{n}=1-15)$ clusters with temperature and molecular number are shown in tables 2 and 3. These thermodynamic properties with temperature and their characteristics can be used to explore the thermodynamic problems of the evolution of deoxidation products in the process of yttrium deoxidation chemical reaction.

\section{Conclusions}

The initial configuration of Yttrium oxide clusters $\left(\mathrm{Y}_{2} \mathrm{O}_{3}\right)_{n}(\mathrm{n}=1-15)$ was creatively constructed by combining artificial bee colony algorithm with density functional theory. The structures of large and medium-sized yttrium oxide clusters with molecular number greater than 10 were established for the first time, and their ground state structures were determined by structural optimization and frequency analysis in Gaussian 09 software package. The average binding energy, second-order difference energy, H-L energy gap,density of states and thermodynamic properties of each structure were calculated and analyzed in detail.the result shows:

1. For small clusters with $\mathrm{n}<5$, oxygen atoms and yttrium atoms tend to form cage-like clusters. With the increase of size $(n=6-15)$, the clusters structure change from cage-like to space stair-like, and gradually evolves into stable ellipsoid-like structure.

2. The stability and molecular orbital of the clusters were analyzed in detail, and it was found that the cluster structure was generally stable. Because the valence bond orbital of oxygen atom is less than half full $\left(2 \mathrm{P}^{4}\right)$, and the valence bond orbital of yttrium atom is full $\left(5 \mathrm{~S}^{2}\right)$, the probability of electron filling $\mathrm{p}$ orbital is much larger than that of filling s orbital, so the binary mixed yttrium oxide clusters have more stable structures than the single yttrium clusters. When the number of cluster molecules $\mathrm{n}=2,4,7,9$, the relative stability is higher, and the second-order energy difference score is basically consistent with the results of H-L energy gap in determining the stability of cluster structure. The major contribution from $\mathrm{S}$ basis function of oxygen (magenta curve) is due to low-lying MOs instead of frontier MOs. HOMO is almost purely contributed by yttrium orbitals.

3. $\mathrm{C}_{\mathrm{p}}, \mathrm{H}$ and $\mathrm{S}$ of $\left(\mathrm{Y}_{2} \mathrm{O}_{3}\right)_{\mathrm{n}}$ increased with the increase of $\mathrm{T}$, and increased with the increase of cluster size. $\mathrm{G}_{\mathrm{v}}$ decreases with the increase of temperature, and the change rule with cluster size is greatly affected by $T$. In the temperature range of $300 \mathrm{~K}-500 \mathrm{~K}$, the $\mathrm{G}_{\mathrm{v}}$ lines intersect each other, indicating that the stability of nanoclusters has changed in the temperature range of 300K-500K. The thermodynamic properties of $\left(\mathrm{Y}_{2} \mathrm{O}_{3}\right)_{n}$ vary greatly with the number of clusters, which is similar to the size effect of the thermodynamic properties of nanoscale materials.

The structure and related thermodynamic data of nanoclusters calculated in this study have certain guiding significance for studying the nucleation of yttrium oxide crystals from the perspective of nanothermodynamics.

\section{Acknowledgement}

The authors gratefully acknowledge the financial support from the National Natural Science Foundation of China(No.51974139) and Jiangxi Province Major Scientific and Technological Research and Development Special Funding Project(No.20194ABC28011).

\section{References}


[1] Cai M H, Ding H, Zhang J S,Li L. Effect of silicon and prior deformation of austenite on isothermal transfor- mation in low carbon steels[J]. Acta Metallurgica Sini- ca (English Letters), 2009, 22(2):100-109.[J].

[2] Li H Z, Liu H T, Wang X L, Cao J M, Li C J, Liu Z Y, Wang G D. Effect of cerium on the as-cast microstructure and tensile ductility of the twin-roll casting Fe- 6. 5wt\% Si alloy[ J]. Materials Letters, 2016, 165,58. [J].

[3] Kang S K, Gow K V. Mechanical properties of rare earth metal treated rail steels[J]. Metallurgical Transactions A, 10(1979)1800-1802.

[4] Smirnov L A, Rovnushkin V A, Oryshchenko A S, et al. Modification of Steel and Alloys with Rare-Earth Elements. Part 1[J]. Metallurgist, 59(2016), 11,p.1053-1061.

[5] Qin J, Liu D F, Zhang Y H, Lai C B. Application sta- tus and development prospect of rare earth in electrical steels[ J]. Journal of Iron and Steel Research, 2018, 30(3):163-170.[J].

[6] Yu X, Zhang Z H, Xie J X. Effects of rare earth ele- ments doping on ordered structures and ductility im- provement of Fe-6. 5wt\%Si alloy[J]. Materials Letters, 2016, 184,294-297.[J].

[7] Zhao Xinyu, Zhang Yu. Synthesis of rare earth ultrafine powders by spray pyrolysis (I) Particle morphology and formation mechanism of Y2O3 powder[J]. Chemical Journal of Chinese Universities. 1998(04): $3-5 .[\mathrm{J}]$.

[8] Hou X, Zhou S, Li W, et al. Study on the effect and mechanism of zirconia on the sinterability of yttria transparent ceramic[J]. Journal of the European Ceramic Society, 30(2010), 15,p.3125-3129.[J].

[9] Low Dimensional Structures; Istanbul Technical University Details Findings in Low Dimensional Structures (A Straightforward Approach for the Synthesis of Nanostructured Y2o3 Particles: Synthesis, Morphology, Microstructure and Crystal Imperfection) [J]. Journal of Physics Research, (2020).[J].

[10] Chen, P.-L. and Chen, I.-W. (1996), Grain Boundary Mobility in Y2O3: Defect Mechanism and Dopant Effects. Journal of the American Ceramic Society, 79: 1801-1809.[J].[J].

[11] Dai B, Deng K, Yang J. A theoretical study of the Y4O cluster[J].Chemical Physics Letters , 364(2002), 1,p.188-195.

[12] Gu G, Dai B, Ding X, et al. A theoretical study of the Y3O clusters[J]. The European Physical Journal D - Atomic, Molecular, Optical and Plasma Physics , 29(2004).

[13] Yang Z, Xiong S. Structural, electronic, and magnetic properties of $\mathrm{YnO}(\mathrm{n}=2-14)$ clusters: Density functional study[J]. The Journal of Chemical Physics , 129(2008), 12,p.124308.

[14] Yang Z, Xiong S. Structural, electronic and magnetic properties of small yttrium trioxide clusters from density functional calculations[J].Journal of Physics B Atomic Molecular Physics , 42(2009)245101.

[15] Wu H, Wang L. Photoelectron Spectroscopy and Electronic Structure of ScOn- $(\mathrm{n}=1-4)$ and YOn$(\mathrm{n}=1-5)$ : Strong Electron Correlation Effects in ScO-and YO-[J]. The Journal of Physical Chemistry A, 102(1998), 46,p.9129-9135.[J].

[16] Pramann A, Nakamura Y, Nakajima A, et al. Photoelectron Spectroscopy of Yttrium Oxide Cluster Anions: Effects of Oxygen and Metal Atom Addition[J]. The Journal of Physical Chemistry A, 105(2001), 32,p.7534-7540.[J].

[17] Knickelbein M. Photoionization Spectroscopy of Yttrium Clusters: Ionization Potentials for Yn and $\mathrm{YnO}(\mathrm{n}-231)[\mathrm{J}]$. J.Chem.Phys, (1995).[J].

[18] Rahane A B. Density Functional Calculations of the Structural and Electronic Properties of (Y2O3)n0, \pm 1 Clusters with $\mathrm{n}=1-10[\mathrm{~J}]$. The Journal of Physical Chemistry. $[\mathrm{J}]$. 
[19] Qiyao Z, Longjiu C. Structural Determination of (Al2O3)(n) (n = 1-15) Clusters Based on Graphic Processing Unit.[J]. Journal of chemical information and modeling, 55(2015), 5,p.[J].

[20] Rong Li L C. Structural determination of $(\mathrm{Al} 2 \mathrm{O} 3) \mathrm{n}(\mathrm{n}=1-7)$ clusters based on density functional calculation $[\mathrm{J}] .(2012) \cdot[\mathrm{J}]$.

[21] Xiao Jianyun, Tan Peng. Density functional study on the structure and properties of (HgSe)n(n=1-6) clusters[J]. Journal of Atomic and Molecular Physics, 2015, 32(01) : 79-85.[J].

[22] M. J. Frisch, G. W. Trucks, H. B. Schlegel, G. E. Scuseria, M. A. Robb, J. R. Cheeseman, G. Scalmani, V. Barone, G. A. Petersson, H. Nakatsuji, X. Li, M. Caricato, A. V. Marenich, J. Bloino, B. G. Janesko, R. Gomperts, B. Mennucci, H. P. Hratchian,J. V. Ortiz, A. F. Izmaylov, J. L. Sonnenberg, D. WilliamsYoung,F. Ding, F. Lipparini, F. Egidi, J. Goings, B. Peng, A. Petrone,T. Henderson, D. Ranasinghe, V. G. Zakrzewski, J. Gao, N. Rega,G. Zheng, W. Liang, M. Hada, M. Ehara, K. Toyota, R. Fukuda,J. Hasegawa, M. Ishida, T. Nakajima, Y. Honda, O. Kitao, H. Nakai,T. Vreven, K. Throssell, J. A. Montgomery, Jr., J. E. Peralta,F. Ogliaro, M. J. Bearpark, J. J. Heyd, E. N. Brothers, K. N. Kudin,V. N. Staroverov, T. A. Keith, R. Kobayashi, J. Normand,K.Raghavachari, A. P. Rendell, J. C. Burant, S. S. Iyengar,J. Tomasi, M. Cossi, J. M. Millam, M. Klene, C. Adamo, R. Cammi,J. W. Ochterski, R. L. Martin, K. Morokuma, O. Farkas, J. B.Foresman, and D. J. Fox, Gaussian, Inc., Wallingford CT, 2016.[J][J].

[23] Zhang J, Dolg M. ABCluster: the artificial bee colony algorithm for cluster global optimization[J]. Physical Chemistry Chemical Physics, 17(2015), 37,p.24173-24181[J].

[24] Zhang J, Dolg M. Global optimization of clusters of rigid molecules using the artificial bee colony algorithm[J]. Physical Chemistry Chemical Physics, 18(2016), 4,p.3003-3010.[J].

[25] Zhang J, Glezakou V, Rousseau R, et al. NWPEsSe: An Adaptive-Learning Global Optimization Algorithm for Nanosized Cluster Systems[J]. Journal of Chemical Theory and Computation, 16(2020), 6,p.39473958. [J].

[26] Cao X, Dolg M. Relativistic Pseudopotentials[M]. Relativistic Methods for Chemists, Barysz M, Ishikawa Y, Dordrecht:Springer Netherlands, 2010,p.215-277.[J].

[27] Mclean A D, Chandler G S. Contracted Gaussian basis sets for molecular calculations. I. Second row atoms, $\mathrm{Z}=11-18[\mathrm{~J}]$. The Journal of Chemical Physics, 72(1980), 10,p.5639-5648.[J].

[28] Dolg M, Stoll H, Savin A, et al. Energy-adjusted pseudopotentials for the rare earth elements[J]. Theoretica chimica acta, 75(1989), 3,p.173-194.[J].

[29] Xun-Lei D, Wei X, Yan-Ping M, et al. Density functional study on cage and noncage (Fe2O3)n clusters.[J]. The Journal of chemical physics, 130(2009), 1,p.[J].

[30] Li X, Wang H, Lv R, et al. Correlations of the Stability, Static Dipole Polarizabilities, and Electronic Properties of Yttrium Clusters[J]. The Journal of Physical Chemistry A , 113(2009), 38,p.10335-10342.

[31] Wang J L W G H Z. Physcial Review B[J]. 66(2002), 3,p.[J].

[32] Zeyu Liu, Tian Lu, Qinxue Chen, An sp-hybridized all-carboatomic ring,

cyclo[18]carbon: Electronic structure, electronic spectrum, and optical nonlinearity,

Carbon, 165, 461-467 (2020) DOI: 10.1016/j.carbon.2020.05.023[J].

[33] Tian Lu, Feiwu Chen, Multiwfn: A Multifunctional Wavefunction Analyzer, J. Comput. Chem. 33, 580-592 (2012) DOI: 10.1002/jcc.22885[J].

[34] Lu, Tian; chen, qinxue (2020): Shermo: A General Code for Calculating Molecular Thermochemistry Properties. ChemRxiv. Preprint. https://doi.org/10.26434/chemrxiv.12278801.v1 [J]. 
[35] Wang G C, Wang Q, Li S L, et al. A Multi-step Thermodynamic Model for Alumina Formation during Aluminum Deoxidation in Fe-O-Al Melt[J].Acta Metallurgica Sinica (English Letters), (2015).

[36] Wang G C, Wang Q, Li S L, et al. Evidence of multi-step nucleation leading to various crystallization pathways from an Fe-O-Al melt[J].Scientific reports , 4(2014)5082.

\begin{tabular}{|c|c|c|c|c|c|}
\hline$\left(\mathbf{Y}_{2} \mathbf{O}_{3}\right)_{n}$ & 1 & 2 & 3 & 4 & 5 \\
\hline $\mathbf{A}$ & $\mathbf{1 A}, \mathrm{D}_{3 \mathrm{~h}}, \mathbf{0 . 0 0}$ & $2 A, T_{d}, 0.00$ & $3 \mathrm{~A}, \mathbf{C}_{1}, 0.00$ & 4 & $5 A, C_{2 v}, 0.00$ \\
\hline B & $1 \mathrm{~B}, \mathrm{C}_{2 \mathrm{v}}, 1.56$ & $2 B, C_{2 h}, 1.45$ & 3B,Cs,0.07 & $4 B, C_{2 v}, 0.64$ & $5 B, C_{1}, 1.78$ \\
\hline C & $1 C, D_{\text {oh }}, 4.10$ & $2 \mathrm{C}, \mathrm{C}_{2 \mathrm{~h}}, \mathrm{O} .30$ & $3 \mathrm{C}, \mathrm{C}_{\mathrm{S}}, 0.08$ & $4 \mathrm{C}, \mathrm{C}_{1}, 0.48$ & $5 C, C_{1}, 2.12$ \\
\hline D & & & $\begin{array}{c}3 \mathrm{D}, \mathrm{C}_{2 \mathrm{v}}, 0.45 \\
0\end{array}$ & $4 \mathrm{D}, \mathrm{C}_{1}, 2.56$ & $5 D, C_{1}, 2.39$ \\
\hline $\mathbf{E}$ & & & $3 \mathrm{E}, \mathrm{D}_{3 \mathrm{~h}}, 0.79$ & $4 E, D_{3 h, 3.80}$ & 5E, C2v, 2.68 \\
\hline
\end{tabular}




\begin{tabular}{|c|c|c|c|c|c|}
\hline$\left(\mathbf{Y}_{2} \mathrm{O}_{3}\right)_{n}$ & 6 & 7 & 8 & 9 & 10 \\
\hline $\mathbf{A}$ & 6А, C, 0.00 & 7A,CS,0.00 & 8A,CS,0.00 & 9A,C1,0.00 & \\
\hline B & $6 \mathrm{~B}, \mathrm{C}_{1}, 0.22$ & &  & 9B,C $1,0.12$ & 10B,C $1,0.22$ \\
\hline C & 6C, $, \mathrm{C}_{1}, 0.22$ & 7C,Cs, 0.26 & $8 \mathrm{C}, \mathrm{C}, 0.20$ & $9 \mathrm{C}, \mathrm{C}_{1}, 0.32$ & $\begin{array}{l}685350 \\
10 C, C_{1}, 0.49\end{array}$ \\
\hline D & $6 \mathrm{D}, \mathrm{C}_{1}, 0.27$ & 7D, $C_{1}, 0.52$ & 8D,Cs,, 0.93 & $\begin{array}{r}908 \\
9 D, C_{1}, 0.84\end{array}$ & $10 \mathrm{D}, \mathrm{C}_{1}, 0.87$ \\
\hline $\mathbf{E}$ & $6 E, C_{1}, 0.47$ & $7 \mathrm{E}, \mathrm{C}_{\mathbf{1}}, \mathbf{0 . 6 8}$ & $8 \mathrm{E}, \mathrm{D}_{2 \mathrm{~d}}, 1.16$ & $\begin{array}{c}2,910 \\
0.580 \\
9 E, C 1,1.06\end{array}$ & $10 E, C_{1}, 1.15$ \\
\hline
\end{tabular}




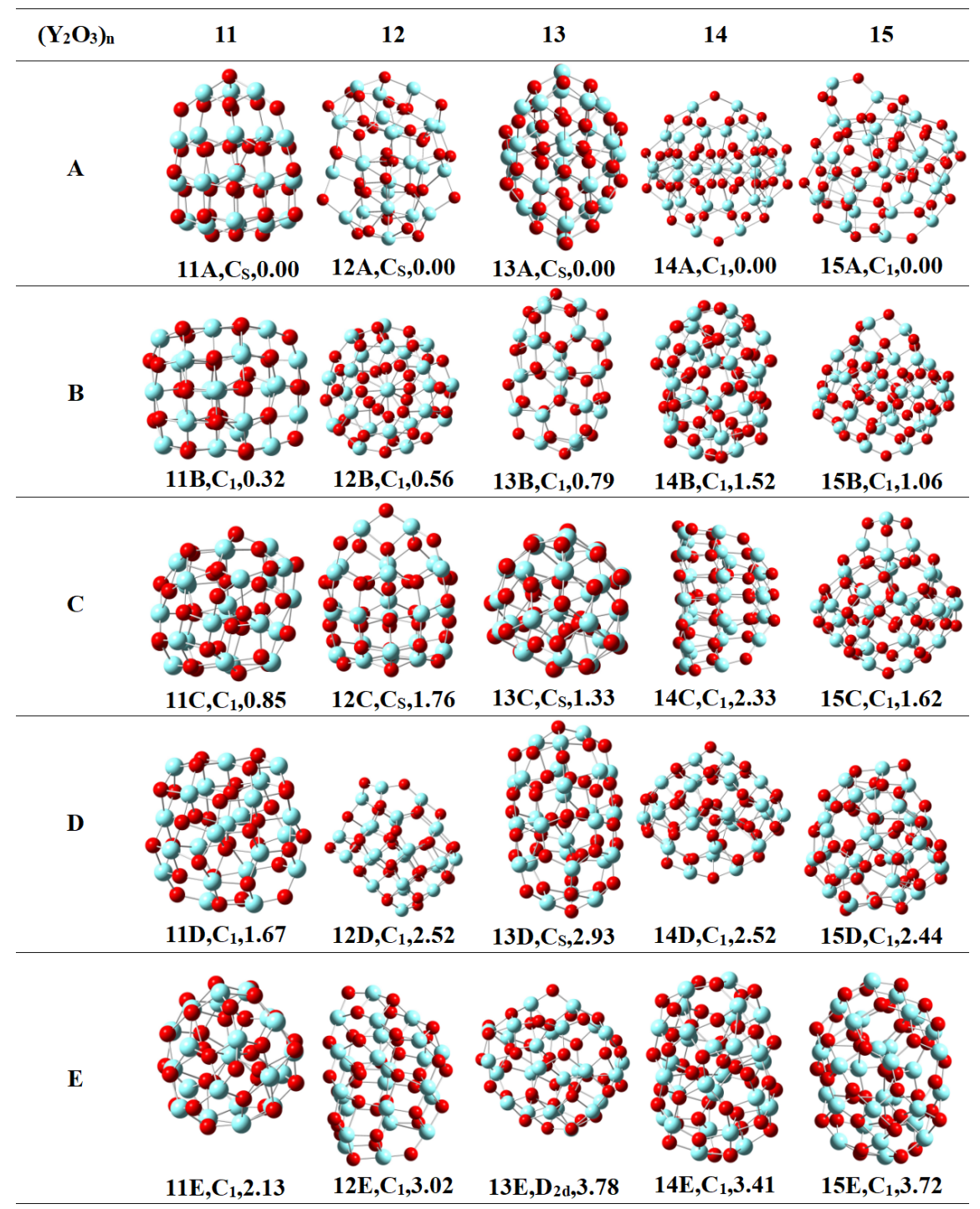



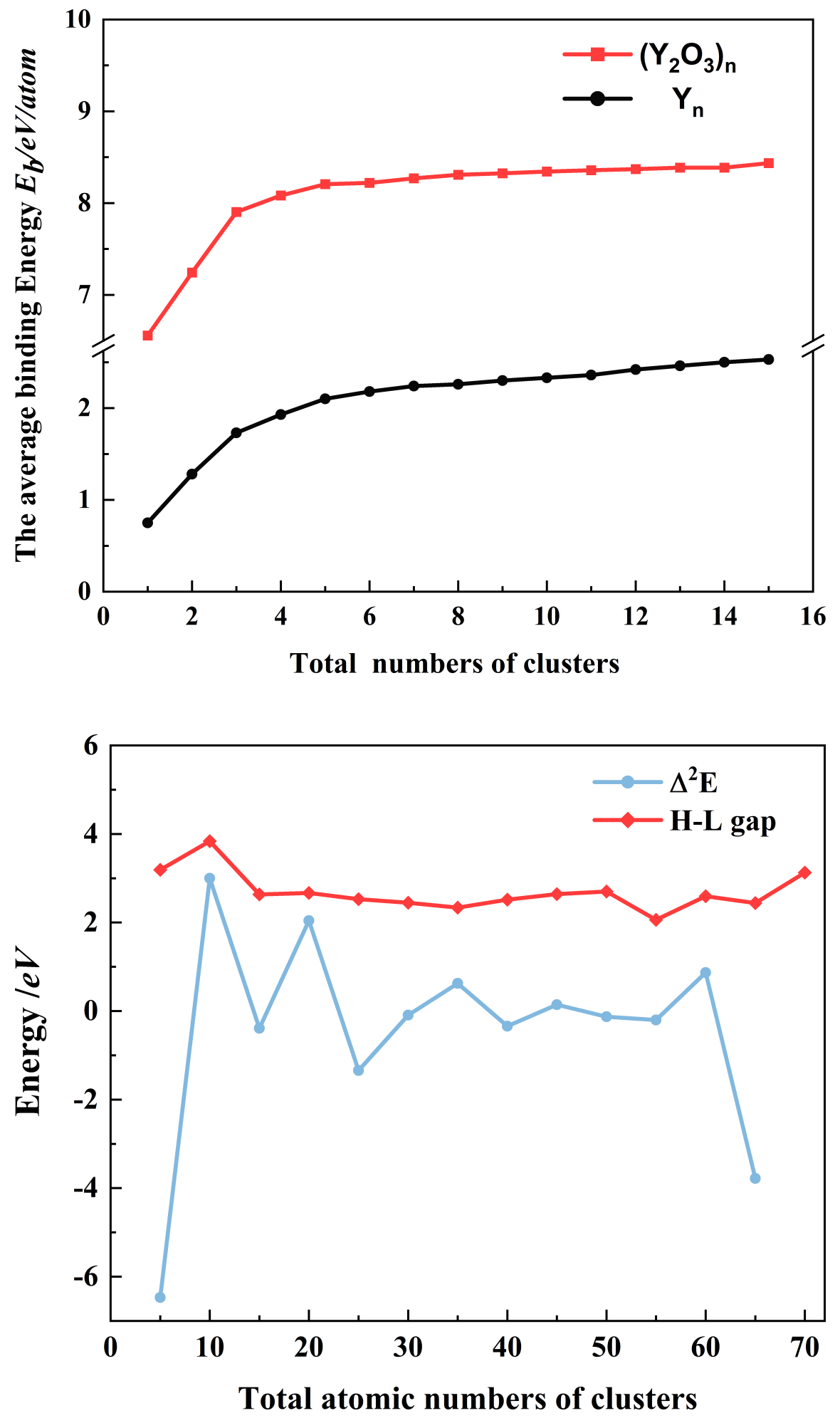

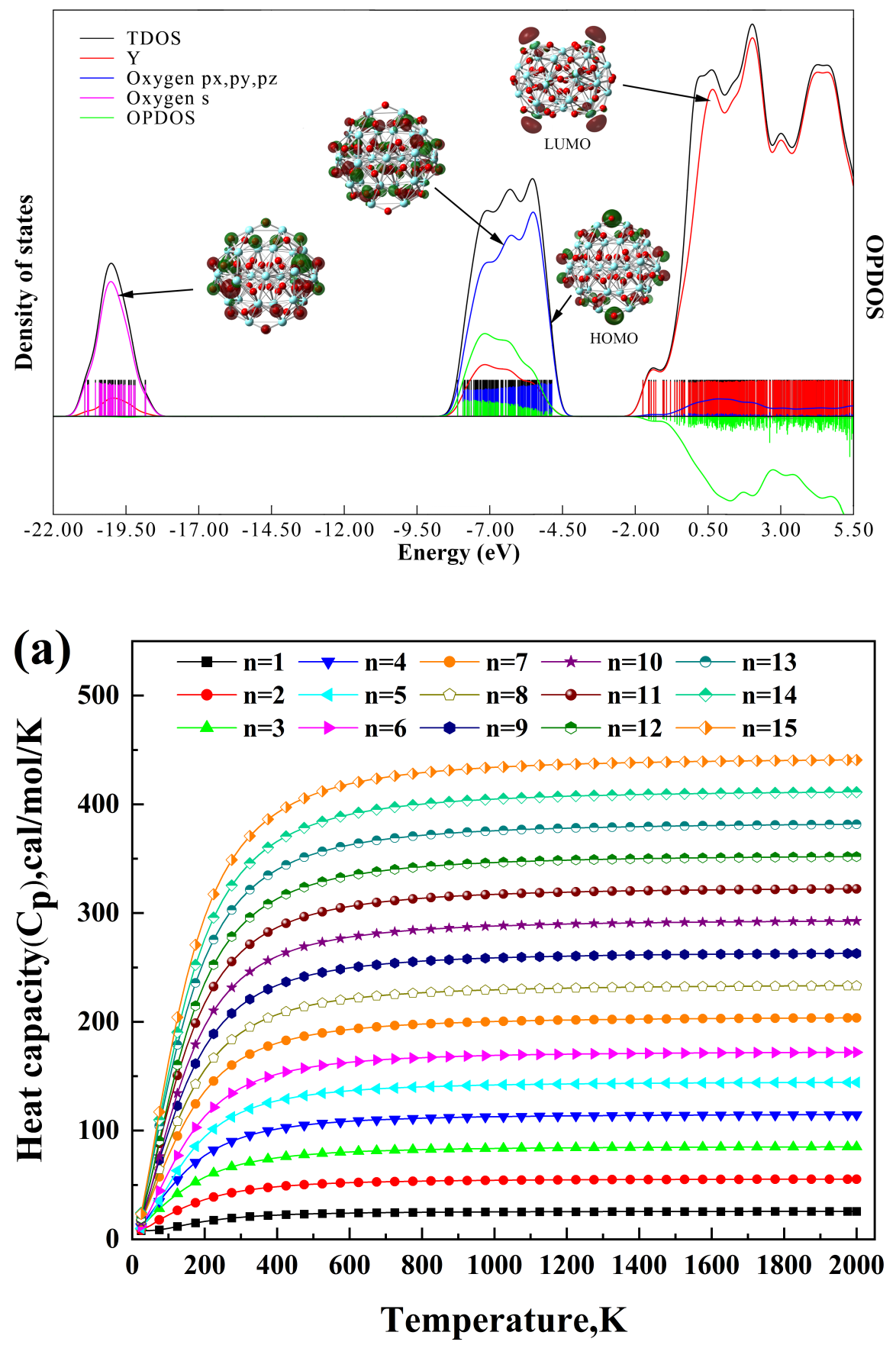

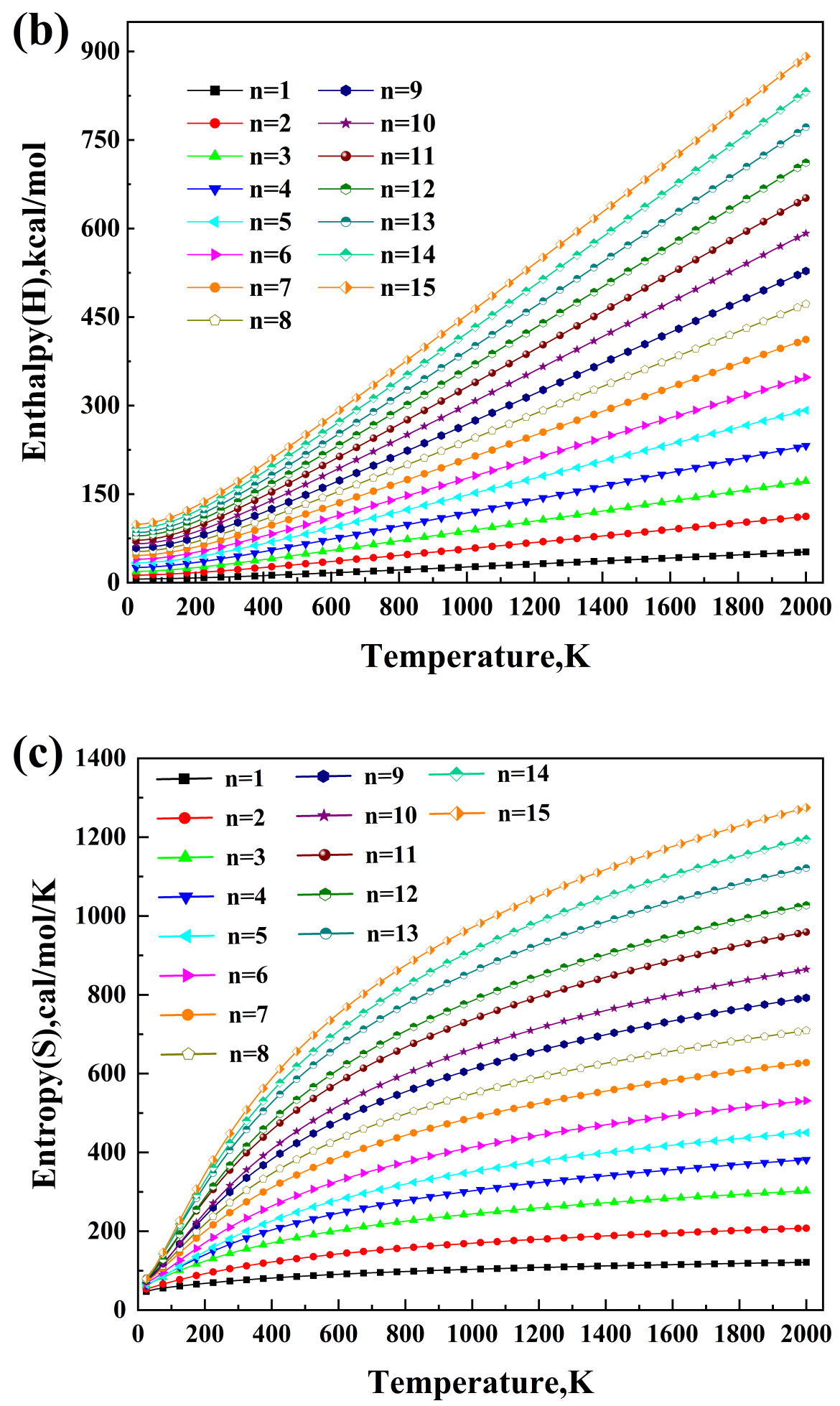

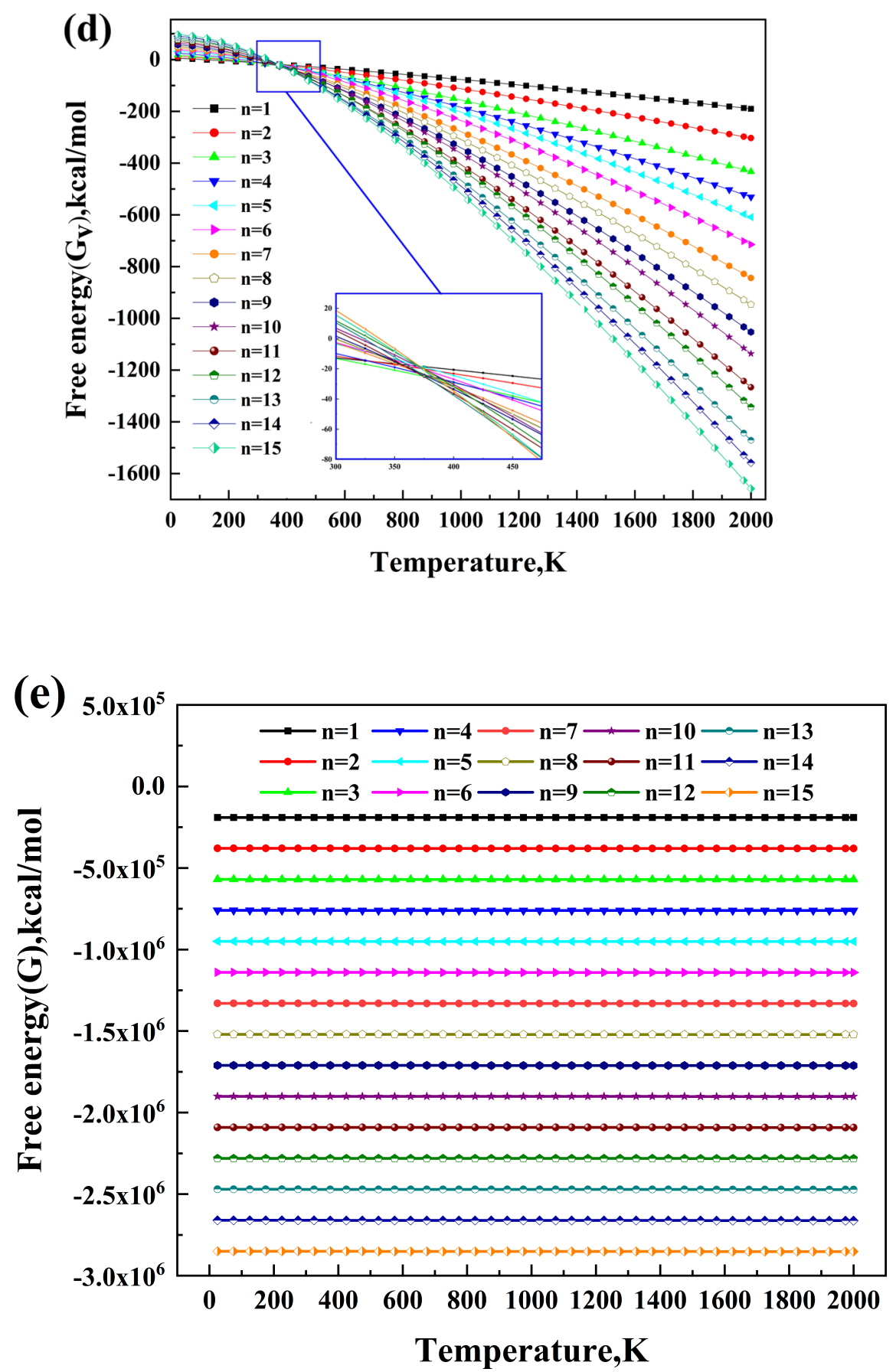

\section{Hosted file}

Table 1.xlsx available at https://authorea.com/users/415723/articles/523537-structuralstability-and-thermodynamic-properties-of-y2o3-n-n-1-15-clusters-based-on-densityfunctional-theory 


\section{Hosted file}

Table 2.xlsx available at https://authorea.com/users/415723/articles/523537-structuralstability-and-thermodynamic-properties-of-y2o3-n-n-1-15-clusters-based-on-densityfunctional-theory

\section{Hosted file}

Table 3.xlsx available at https://authorea.com/users/415723/articles/523537-structuralstability-and-thermodynamic-properties-of-y2o3-n-n-1-15-clusters-based-on-densityfunctional-theory 